\title{
Author Correction: Continuous monitoring of a trapped superconducting spin
}

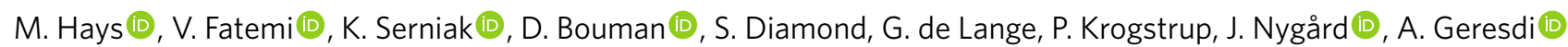
and M. H. Devoret (1D

Correction to: Nature Physics https://doi.org/10.1038/s41567-020-0952-3, published online 6 July 2020.

In this Letter the following original sentence has been amended for clarity: "As the Kramers theorem does not hold in the presence of a non-zero weak-link phase bias $\varphi$, the splitting of the spin states requires an additional ingredient."; it has been changed to: "Although the presence of a non-zero weak-link phase bias $\varphi$ breaks time-reversal symmetry so that the Kramers theorem does not hold, this alone is insufficient to lift the spin degeneracy — an additional effect is required." The online versions of the Letter have been amended.

Published online: 29 June 2021

https://doi.org/10.1038/s41567-021-01309-w

(๑) The Author(s), under exclusive licence to Springer Nature Limited 2021

\section{Author Correction: Strong-interaction matter: Fireball spectroscopy}

\section{Ralf Rapp (1)}

Correction to: Nature Physics https://doi.org/10.1038/s41567-019-0614-5, published online 29 July 2019.

In the version of this News \& Views article originally published, the Acknowledgements section was missing and should have read "This work is supported by the US National Science Foundation under grant no. PHY-1614484.. The online versions of the article have been amended.

Published online: 21 July 2021

https://doi.org/10.1038/s41567-021-01322-Z

() Springer Nature Limited 2021 